\title{
Análisis del grado de fidelidad en la ejecución de un programa de competencia familiar
}

\author{
Carmen Orte Socias \\ Carmen Touza Garma \\ Lluis Ballester Brage \\ UNIVERSITAT DE LES ILLES BALEARS
}

\begin{abstract}
Resumen
Desde el año 2003, el "Grupo de Investigación y Formación Educativa y Social” ' de la Universidad de las Islas Baleares ha estado trabajando en la adaptación y validación a la población española del "Strengthening Families Program” (Kumpfer y DeMarsh, 1985; Kumpfer, DeMarsh y Child, 1989) ${ }^{2}$. El resultado de este trabajo es el "Programa de Competencia Familiar" que hemos aplicado con dos muestras de familias de usuarios de Proyecto Hombre en Palma y sus respectivos grupos control. Tras estas dos aplicaciones es necesario analizar el proceso de ejecución para la optimización de sus componentes (contenidos, materiales, niveles de retención, etc.). En este artículo exponemos la metodología y los resultados que hemos obtenido en una de las dimensiones de esta evaluación: la fidelidad al programa.
\end{abstract}

PALABRAS-CLAVE: prevención, problemas de conducta, consumo de drogas, evaluación, fidelidad.

\section{Summary}

Since 2003 "Grupo de Investigación y Formación Educativa y Social" [1] from Uni- versity of Balearic Islands has been working in the adaptation and validation to the Spanish population of the "Strengthening Families Program" (Kumpfer y DeMarsh, 1985; Kumpfer, DeMash and Child, 1989). The result of this work is the "Programa de Competencia Familiar", that we have implemented with two samples of families from the users of the Proyecto Hombre from Palma and their respective control groups. After these two implementations, it's necessary to analyze the execution process in order to achieve the optimization of its components (contents, material, retention levels...). In this article we expose the methodology and the results that we have obtained in one of the dimensions of this assessment: the fidelity to the program.

KEY WORDS: prevention, conduct disorders, drug abuse, assessment, fidelity.

El programa de Competencia Familiar (PCF) surge de la adaptación y validación en nuestro medio del Programa Stregthening Families Program (SFP), de Kumpfer y DeMarsh (1985), Kumpfer, DeMarsch y Child (1989), y revisiones posteriores de 1998 y 2004. El SFP es un programa que ha demostrado una gran eficacia, en evaluaciones exter- 


\begin{tabular}{|c|c|c|c|}
\hline & Padres y madres & Hijos e hijas & Familias \\
\hline 1 & Introducción y formación del grupo & Saludo y reglas & Introducción y formación del grupo \\
\hline 2 & $\begin{array}{l}\text { Expectativas, desarrollo y manejo } \\
\text { del estrés }\end{array}$ & Habilidades sociales I (escucha activa) & Juego de los hijos e hijas \\
\hline 3 & Recompensas & Habilidades sociales II (conversación) & $\begin{array}{l}\text { Juego de los hijos y de las hijas } \\
\text { (recompensas) }\end{array}$ \\
\hline 4 & Metas y objetivos & Aprender buen comportamiento & Metas y objetivos \\
\hline 5 & $\begin{array}{l}\text { Atención diferencial: (prestar } \\
\text { atención e ignorar) }\end{array}$ & $\begin{array}{l}\text { Cómo decir "NO" y mantenerse } \\
\text { fuera de problemas }\end{array}$ & Atención diferencial \\
\hline 6 & Comunicación I (mejorar las relaciones) & Comunicación I (mejorar las relaciones) & $\begin{array}{l}\text { Comunicación I (introducción al juego } \\
\text { familiar) }\end{array}$ \\
\hline 7 & Comunicación II (reuniones familiares) & Comunicación II (reuniones familiares) & $\begin{array}{l}\text { Comunicación II (consolidación del } \\
\text { juego familiar) }\end{array}$ \\
\hline 8 & D rogas y familia (factores de riesgo) & Alcohol y drogas & Aprender de los padres y de las madres \\
\hline 9 & Resolver problemas y dar instrucciones & Resolver problemas & Resolver problemas y dar instrucciones \\
\hline 10 & $\begin{array}{l}\text { Poner límites I (reconducir el mal } \\
\text { comportamiento) }\end{array}$ & $\begin{array}{l}\text { Introducción al Juego de los Padres } \\
\text { y de las Madres }\end{array}$ & El Juego de los Padres y de las Madres I \\
\hline 11 & Poner límites II (practicar) & $\begin{array}{l}\text { Habilidades de afrontamiento I } \\
\text { (reconocer sentimientos) }\end{array}$ & $\begin{array}{l}\text { El Juego de los Padres } \\
\text { y de las Madres II }\end{array}$ \\
\hline 12 & $\begin{array}{l}\text { Poner límites III (resolver problemas } \\
\text { de comportamiento) }\end{array}$ & $\begin{array}{l}\text { Habilidades de afrontamiento II } \\
\text { (afrontar la crítica) }\end{array}$ & $\begin{array}{l}\text { El Juego de los Padres } \\
\text { y de las Madres III }\end{array}$ \\
\hline 13 & $\begin{array}{l}\text { Crear y utilizar programas de } \\
\text { comportamiento }\end{array}$ & $\begin{array}{l}\text { Habilidades de afrontamiento III } \\
\text { (manejar el enfado) }\end{array}$ & $\begin{array}{l}\text { Recordar lo que nos ayuda y por qué } \\
\text { nos ayuda }\end{array}$ \\
\hline 14 & Mantener el buen comportamiento & Graduación, recursos y repaso & Final del programa graduación \\
\hline
\end{tabular}

nas e independientes al equipo que lo originó (Center for Substance Abuse Prevention, 1998; Biglan et al. 2003), para obtener cambios en los factores de protección y de riesgo relacionados con la aparición y el desarrollo de problemas de conducta, incluyendo el consumo de alcohol y/o drogas.

Entre los principales factores protectores nos encontramos varios relacionados con el ejercicio adecuado de la parentalidad: las relaciones positivas padres-hijos, la utilización de métodos de disciplina positivos; el control y la supervisión de los padres y la comunicación familiar de valores y expectativas saludables y positivas (Ary et al. 2000; CSAP, 2000). Como indica Kumpfer (1999), el apoyo parental es el factor protector más impor- tante, ayuda a los hijos a desarrollar sueños, propósitos y metas en la vida.

El SFP y PCF (Orte y GIFES, 2005a, 2005b; Orte y GIFES, 2006a) son programas multicomponente porque combinan un curriculum de habilidades sociales y de vida dirigido a los niños y jóvenes, un curriculum de entrenamiento parental conductual y un curriculum centrado en la familia, que integra las habilidades aprendidas por los hijos y las habilidades aprendidas por los padres. Con este tipo de programas multicomponentes el tamaño de los efectos alcanzados es mucho mayor que el que se obtiene utilizando cualquiera de los otros tres tipos de programas de forma individual (Tobler y Kumpfer, 2000). También la retención es mucho 
más elevada cuando se utiliza este tipo de modalidad. Como señala Kumpfer (2003), y hemos podido también constatar, una de las razones es el interés de los hijos, que animan a los padres a continuar en el programa. A lo largo de 14 sesiones y después de una merienda común, la familia se divide en grupos: en la primera hora, el grupo de padres y el grupo de hijos aprenden y practican los contenidos y las habilidades en cada uno de sus grupos. En la segunda hora las familias se reúnen para practicar juntos las habilidades que unos y otros han aprendido en la primera hora. Los contenidos de las sesiones aparecen recogidos en la tabla 1. Las sesiones han sido conducidas por terapeutas de Proyecto Hombre con una amplia experiencia en el manejo de grupos, en el trabajo con población en proceso de desintoxicación y, además, especialmente formados para la aplicación del programa.

\section{Justificación y Objetivos}

Hemos realizado dos aplicaciones del programa. Finalmente la muestra obtenida está constituida por 58 adultos ( 28 en los grupos experimentales y 30 en los controles). Son hombres y mujeres que asistían a programas de tratamiento por abuso de cocaína o son sus parejas y con una media de edad de 39 años. En cuanto a los niños, han participado un total de 35 (19 en los grupos experimen tales y 16 en los controles), con una media de 10,6 años (el rango de edades era de 6 a 14 años). En total se trata de 31 familias (15 en los grupos experimentales y 16 en los controles). Todos los participantes en los grupos experimentales residían en Mallorca, mientras que los controles los hacían en Barcelona, Córdoba, Sevilla y Alicante.

Tras esta dos aplicaciones hemos analizado los datos que fuimos recogiendo para evaluar el proceso de ejecución del programa. Esta evaluación nos permitió detectar dificultades en los participantes, en el programa o en su ejecución. También nos ha sido muy útil para obtener información que permita mejorar los contenidos, los materiales y el proceso de ejecución (Orte y Touza, 2006) y, más adelante, nos ayudará a vincular los resultados con el proceso formativa Un aspecto fundamental de este tipo de evalua ción es conocer el grado de fidelidad al programa, en qué medida la ejecución real del programa se ajustó a sus componentes (contenidos y actividades) y si ha habido varia ciones entre sesiones y entre subprogramas (padres y madres, hijos e hijas y familias). Estos son los objetivos que nos hemos planteado en este estudio. En los siguientes apartados mostramos la metodología y los resultados que hemos obtenido.

\section{Metodología}

\subsection{Sujetos}

Como explicamos en el siguiente apartado, la información necesaria para evaluar la fidelidad al programa la hemos obtenido a partir de cuestionarios y entrevistas con los forma-

\begin{tabular}{|lccc|}
\hline \multicolumn{4}{|c|}{ TABLA 2. TIPO DE FORMADOR QUE DIRIGIÓ LAS SESIONES Y LAS EVALUÓ } \\
\hline Formadores habituales & $\begin{array}{c}\text { 1 Formador habitual } \\
\mathbf{+ 1} \text { Formador sustituto }\end{array}$ & Total \\
\hline Padres/Madres & $85,7 \%$ & $14,3 \%$ & $100 \%$ \\
\hline Hijos/Hijas & $89,3 \%$ & $10,7 \%$ & $100 \%$ \\
\hline Familias & $79,9 \%$ & $23,1 \%$ & $100 \%$ \\
\hline
\end{tabular}


dores. En cada aplicación han participado cuatro formadores habituales y dos forma dores sustitutos, cuando los primeros no podían asistir a alguna sesión. Todos habían sido formados en la fundamentación y ejecución del programa. Como muestra la siguiente tabla, la mayoría de las sesiones han sido dirigidas por los formadores habituales, que, por lo tanto, han sido la principal fuente de información de la que obtener los datos que analizaremos más adelante.

En las sesiones familiares aparece el porcentaje más elevado de sesiones en las que hubo un formador sustituto. Este resultado es lógico si pensamos que estas sesiones fueron dirigidas por los cuatro formadores, los dos de las de padres y los dos de las de hijos, de forma que cuando ha habido un formador sustituto en alguna de ellas, también ha estado en la de familias.

\subsection{Instrumentos y procedimiento}

Para la evaluación del proceso de ejecución del programa hemos utilizados varios instrumentos (Orte et al. 2006b; Orte y Touza, 2006), entre ellos el "Cuestionario de fidelidad de la sesión". Este cuestionario se cumplimentó al finalizar las sesiones 2, 3, 4, 10, 11 y 12 de padres, hijos y familias. En él podemos distinguir cuatro apartados. El primero sobre los formadores y participantes en la sesión, así como sobre su duración. El segundo apartado se cumplimenta de forma conjunta por los formadores y valora el espacio y materiales utilizados y el grado de fidelidad en el desarrollo de la sesión. En el tercer apartado cada formador se autoevalúa y valora al grupo. Finalmente, se plantean preguntas abiertas que son contestadas de forma conjunta por los formadores de cada una de las sesiones a través de una entrevista hecha por los responsables de la evaluación.

Para evaluar el grado de fidelidad, los formadores tienen que informar si han desarrollado o no cada uno de los componentes de la sesión (explicaciones, debates y actividades) y valorarlo en una escala de tres grados.

\section{Análisis e interpretación de los resultados}

La primera pregunta que nos hacemos es el nivel de fidelidad al programa. Las figuras 1 y 2 muestran los porcentajes de fidelidad alcanzados sesión a sesión en cada subprograma y en cada aplicación. Los porcentajes son elevados pero necesitamos saber si se han producido diferencias en el grado de fidelidad entre aplicaciones en los tres subprogramas. Las figuras parecen reflejar un mayor grado de fidelidad en la segunda aplicación frente a la primera, pero ¿son significativas estas diferencias? Los resultados al comparar los porcentajes medios de fidelidad en los subprogramas de las dos aplicaciones nos indican que se ha obtenido el mismo grado de fidelidad en las sesiones de padres y madres $(\mathrm{t}=-1,804 ; \mathrm{p}=0,122)$ y de hijos e hijas $(t=-1,490 ; p=0,186)$, mientras que en la segunda aplicación del subprograma de familias el nivel de fidelidad ha sido significativamente superior al alcanzado en la primera aplicación ( $\mathrm{t}=-2,758$; 0,020).

Otro aspecto que parece reflejarse en la

FIGURA 1. PORCENTAJES DE FIDELIDAD EN LAS SESIONES DE LA PRIMERA APLICACIÓN

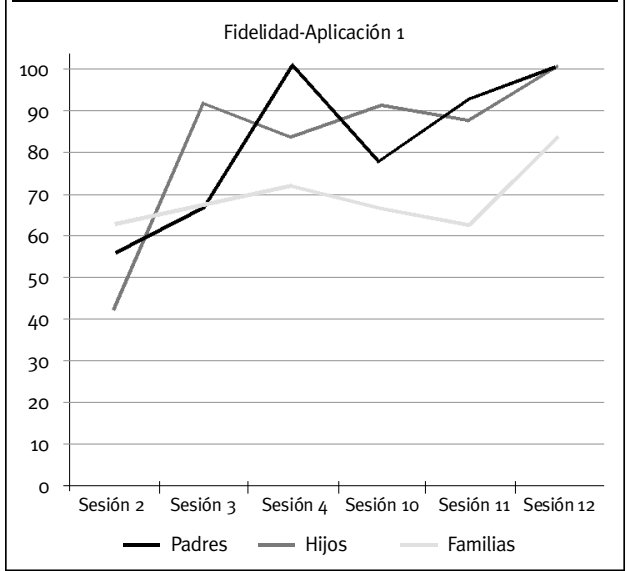




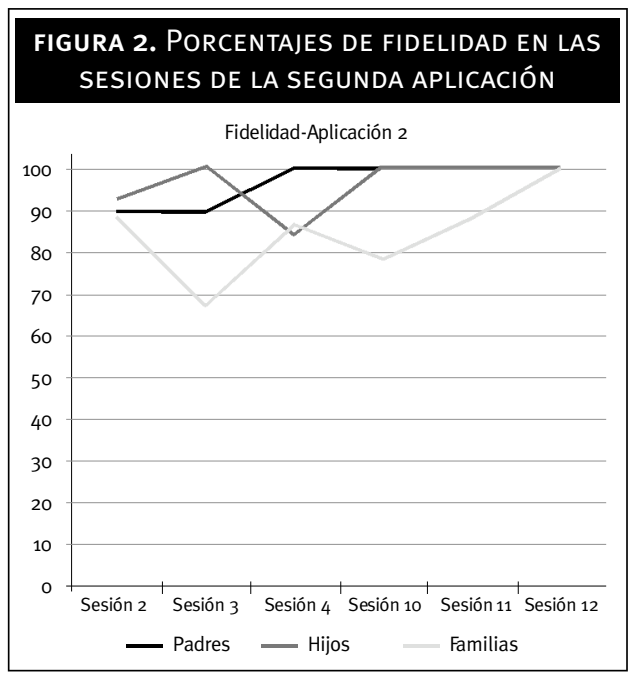

figuras anteriores es que, en ambas aplicaciones, el grado de fidelidad alcanzado en las sesiones familiares es menor que el logrado en los otros dos subprogramas. Los análisis estadísticos que hemos realizado confirman esta observación. Mientras que no hemos encontrado diferencias significativas entre el programa de padres y madres y el de hijos e hijas ( $t=0,000 ; p=1,000$ ), sí que las encontramos al comparar las sesiones familiares con las de padres y madres $(t=2,266 ; p=0,034)$, y con las de los niños ( $t=2,152 ; \mathrm{p}=0,043)$.

Las sesiones de los tres subprogramas están compuestas de tres tipos de elementos: explicaciones (sobre los contenidos), debates (sobre los contenidos y sobre la realización de las tareas para casa) y actividades (role play, juegos, etc.). Nos interesaba saber si habían "funcionado igual de bien" en ambas aplicaciones en lo que se refiere a la ampli- tud y la profundización en los contenidos, al grado de participación y al refuerzo de los formadores a los participantes. Como recoge la tabla 3, los resultados muestran que no se han producido diferencias en ninguno de los componentes, además las valoraciones hechas por los formadores han obtenido medias bastante elevadas si tenemos en cuenta que se valoraban en una escala de tres grados.

A pesar de que no se hubieran producido diferencias entre aplicaciones, podrían haberse producido entre los diferentes subprogramas, por ejemplo, podríamos pensar que los debates o las explicaciones se habrían desarrollado de una forma más adecuada con los adultos, que con los niños o en las sesiones de familias. Para analizar esta posibilidad, hemos comparado las explicaciones, los debates y las actividades de los tres subprogramas.

Los resultados muestran que las valoraciones medias de las actividades son algo inferiores para las sesiones familiares (media= $1,765)$, frente a las de los padres y madres $($ media $=2,250)$ y las de los niños $($ media $=2,52)$. Sin embargo, estas diferencias no son en ningún caso significativas cuando comparamos las actividades de las sesiones de los adultos, frente a las de los niños $(t=0,193, p=0,849)$ y a las de las sesiones de familia $(t=1,042 ; p=0,311)$; y las actividades de los niños, frente a las de las familias $(\mathrm{t}=0,937, \mathrm{p}=0,359)$.

Los resultados sobre las explicaciones y los debates son muy similares. Las valora ciones medias sobre las explicaciones son elevadas (2,520 en los padres; 2,833 en los hijos y 2,637 en las familias) y tampoco se produ-

\begin{tabular}{|c|c|c|c|c|c|c|c|c|c|}
\hline & \multicolumn{3}{|c|}{ Primera aplicación } & \multicolumn{3}{|c|}{ Segunda aplicación } & \multirow[b]{2}{*}{$\mathbf{t}$} & \multirow[b]{2}{*}{ gl } & \multirow[b]{2}{*}{$\mathbf{p}$} \\
\hline & $\mathbf{N}$ & Media & Desviación & $\mathbf{N}$ & Media & Desviación & & & \\
\hline Explicaciones & 15 & 2,706 & 0,556 & 15 & 2,605 & 0,593 & 0,482 & 28 & 0,634 \\
\hline Debates & 17 & 2,260 & 0,918 & 17 & 2,232 & 0,871 & 0,091 & 32 & 0,928 \\
\hline Actividades & 16 & 2,258 & 1,036 & 16 & 1,805 & 1,005 & 1,257 & 30 & 0,218 \\
\hline
\end{tabular}




\begin{tabular}{|c|c|c|c|c|c|c|}
\hline \multirow[b]{3}{*}{ Sesión y subprograma } & \multicolumn{6}{|c|}{ Medias corregidas } \\
\hline & \multicolumn{3}{|c|}{ Primera aplicación } & \multicolumn{3}{|c|}{ Segunda aplicación } \\
\hline & Actividades & Debates & Explicaciones & Actividades & Debates & Explicaciones \\
\hline 2-Padres/madres & o & 2,66667 & 3 & 3 & 1,33333 & 2 \\
\hline 2-Hijos/hijas & 2,5 & 2,8 & 3 & 1 & 1,2 & 2 \\
\hline 2-Familias & 0,66667 & & 2,2 & 2 & & 3 \\
\hline 3-Padres/madres & 1,5 & 1 & 2,75 & 1,5 & 3 & 3 \\
\hline 3-Hijos/hijas & 3 & 2,6 & & 3 & 2,2 & \\
\hline 3-Familias & 0,66667 & 3 & 2,25 & 0,66667 & 3 & 2,25 \\
\hline 4-Padres/madres & 3 & 2,66667 & 3 & 3 & 2,83333 & 2,5 \\
\hline 4- Hijos/hijas & 3 & 3 & 3 & 0,66667 & 3 & 3 \\
\hline 4-Familias & 3 & 2,4 & 3 & 3 & 1,8 & 3 \\
\hline 10-Padres/madres & & 0,875 & 1 & & 3 & 3 \\
\hline 10-Hijos/hijas & 3 & 3 & 3 & o & 3 & 3 \\
\hline
\end{tabular}

cen diferencias significativas entre ellas al comparar el funcionamiento de las explica ciones desarrolladas con los padres y madres y las de los niños ( $\mathrm{t}=-1,249 ; \mathrm{p}=0,230)$ o las familias $(t=-0,393 ; p=0,699)$; ni entre éstas y las de las sesiones con los niños $(\mathrm{t}=1,103$; $\mathrm{p}=0,286)$.

Los debates también han sido bien valorados, con pequeñas variaciones en sus puntuaciones medias $(2,177$ en las sesiones de los padres y madres; 2,504 en las de sus hijos e hijas y 2,020 en las de las familias). Una vez más, estas diferencias no son significativas. Los debates han funcionado de manera positiva y semejante con los adultos y con los niños $(\mathrm{t}=-1,081 ; \mathrm{p}=0,291)$ o las familias $(t=0,373 ; p=0,713)$, así como entre éstas y el grupo de niños $(t=1,222 ; p=0,236)$.

Pero uno de nuestros objetivos en la evaluación de la ejecución del Programa de Competencia Familiar es obtener información que nos ayude a optimizar su funcionamiento y los resultados. Por eso, consideramos interesante poder analizar las valoraciones que habían hecho los formadores de ambas aplicaciones de las actividades, debates y expli caciones de cada una de las sesiones. Esta información nos indicará qué sesiones y qué componentes debemos revisar. La tabla 4 recoge las medias que hemos obtenido por componentes en cada una de las sesiones de los tres subprogramas y en las dos aplicaciones. Para interpretarla hay que tener en cuenta que los valores pueden oscilar entre o y 3 , reflejando la mínima o la máxima valoración. Para calcularlos hemos multiplicado el número de actividades, debates o explica ciones efectivamente realizados en cada sesión y sus correspondientes valoraciones y lo hemos dividido por el número de actividades, debates o explicaciones que deberían haberse realizado. Cuando en una casilla aparece un cero es porque estaba previsto realizar una actividad, debate o explicación que no llegó a realizarse. Cuando aparece en blanco es porque en esa sesión no está previsto realizar ninguna actividad, debate o explica ción, por lo que la valoración media corregida no se puede calcular.

Las explicaciones han sido muy bien valoradas en todas las sesiones. Las valoraciones más bajas las encontramos en las sesiones 10 y 12 del subprograma de padres y madres, aunque no hay acuerdo entre los formadores de las dos aplicaciones. A pesar de ello, creemos que estos resultados son lógicos por la 
complejidad de los contenidos de ambas. Son sesiones en las que los formadores explican técnicas para poner límites y cómo utilizarlas y, en su opinión, son bastante densas en contenidos. Las comparaciones que hemos realizado entre aplicaciones en las valoracio nes de las explicaciones confirman estas observaciones: no hemos encontrado diferencias significativas en el subprograma de padres y madres $(\mathrm{t}=0,450 ; \mathrm{p}=0,662)$ y tampoco en el de los niños $(t=1,581 ; 0,189)$, ni en las familias ( $\mathrm{t}=-1,332 ; \mathrm{p}=0,231)$

Las valoraciones de los debates son más difíciles de interpretar porque parecen depender más de la aplicación de la que se trate. Los formadores de la segunda aplicación parecen tender a dar valoraciones más bajas al funcionamiento de los debates de las sesiones de hijos e hijas (sesiones 2, 11 y 12) y de familias (sesión 4), mientras que en la primera aplicación podrían haber resultado más complicados los debates con los padres y madres (sesiones 3, 10 y 11). Los resultados de las comparaciones realizadas confirman en parte estas observaciones. Hemos encontrado diferencias significativas en el subprograma de hijos e hijas $(t=2,478 ; p=0,05)$, pero no en el de padres y madres $(t=-1,958, p=0,07)$, ni en el de familias $(t=0,154 ; p=0,882)$. Estas diferencias podrían estar relacionadas con posibles diferencias en las características de los participantes de cada una de las aplicaciones o de los propios formadores.

También los formadores de la segunda aplicación han podido observar más dificultades en el desarrollo de las actividades con los niños (sesiones 2, 4 y 11), mientras que existe acuerdo entre los formadores de ambas aplicaciones en las valoraciones que han hecho de la sesión 3 de padres y madres y de las sesiones 3 y 10 de familias. Estos acuerdos parecen importantes porque la tercera sesión del grupo de padres y madres es una sesión en la que predominan las explicaciones y debates y es posible que quede poco tiempo para las actividades. Algo similar ocu- rre con las sesiones 3 y 10 de familias. Son las sesiones en las que los formadores expli can lo que el programa llama el "Juego de los Hijos y Las Hijas" y el "Juego de los Padres y las Madres", que, entre otras cosas, son el cont exto en el que las familias pondrán en práctica los dos tipos de habilidades que los padres y las madres están aprendiendo: las que tratan de aumentar la frecuencia de las conductas positivas (recompensas), al tiempo que intentan reducir las conductas negativas mediante la atención diferencial, y los métodos para poner límites a las conductas negativas que no pueden ser ignoradas. Son, por lo tanto, dos sesiones en las que las expli caciones y debates pueden dejar menos tiempo para las actividades.

Las comparaciones que hemos realizado apoyan nuestras observaciones sobre las valoraciones de las actividades. Se han producido diferencias significativas en el subprograma de hijos e hijas $(t=3,458 ; p=0,016)$, las valoraciones de las actividades en la primera aplicación son mejores que en la segunda. Sin embargo, no se producen estas diferencias entre aplicaciones en las valoraciones del subprograma de padres y madres $(\mathrm{t}=$ $0,926 ; p=0,390)$, ni en las familias $(t=0,323$; $\mathrm{p}=0,753)$.

\section{Conclusiones}

Los resultados que presentamos en este artículo añaden información al proceso general de evaluación de la ejecución del Programa de Competencia Familiar. Ya habíamos valorado otros aspectos (Orte y Touza, 2006), pero nos quedaba pendiente uno tan importante como es el grado de fidelidad obtenido, ya que, en análisis posteriores, puede ayudarnos a comprender mejor la eficacia del programa. La metodología que hemos utilizado, partir de las valoraciones hechas por los propios formadores, puede tener sus limitaciones, ya que los resultados podrían atribuirse en parte a 
sus propias características. En este sentido es posible plantearse otras metodologías y procedimientos como la grabación de las sesiones y su posterior análisis por observadores externos (Magin et al. s/f), aunque esto supondría otro tipo de dificultades que ahora no podemos abordar. A pesar de ello, creemos que los resultados que hemos obtenido son muy positivos en dos sentidos: nos muestran un adecuado nivel de fidelidad en las dos aplicaciones y nos aportan información muy útil para posteriores aplicaciones. Concretamente, este estudio nos ha permitido sacar las siguientes conclusiones:

Las características de las sesiones familiares en las que hay un mayor número de participantes y se trata de un grupo más heterogéneo, pueden hacer más difícil mantener la fidelidad al programa.

Los distintos componentes de las sesiones (explicaciones, debates y actividades) han funcionado de manera igualmente adecuada con los adultos, con los niños y con las familias.

La cantidad y complejidad de los contenidos de algunas sesiones puede condicionar la fidelidad al programa, sobre todo porque pueden dificultar el desarrollo de las actividades, frente a los debates o las explicaciones.

Las características de los participantes pueden afectar al grado de fidelidad al programa.

\section{Referencias bibliográficas}

Ary, D.V.; Duncan, T. E.; Biglan, A.; Metzler, C.W.; Noell J.W.; Koch, G.: "Development of adolescent problem behavior", en Journal of Abnormal Child Psychology, 27 (2000), pp.141150.

Biglan, A.; Mrazek P. J.; Carnine, D.; Flay, B. R.: "The integration of research and practice in the prevention of youth problem behaviors", en American Psychologist, 58 (2003), pp. 433-440.
Center for Substance Abuse Prevention (1998): "Preventing substance abuse among children and adolescents: family-centeredapproaches", en Prevention Enhancement Protocols System (PEPS). (DHHS Publication No. SMA 3223-FY'98).Washington, D. C: U. S Government Printing Office.

Center for Substance Abuse Prevention (2000): 2000 Annual Summary. Effective prevention principles and programs. Washington, D. C: U. S Government Printing Office.

Kumpfer, K. L. (1999): "Factors and processes contributing to resilience: The resilience framework“, en M. D. Glantz; M. D., Johnson, J. L. (eds.): Resilience and development: positive life adaptations (179-224). New York: Kluwer Academic/Plenum Publishers.

Kumpfer, K. L. (2003): “Selective Prevention Interventions: The Strengthening Families Program", en NIDA Research Monograph 177. Rockville, MD: NIDA.

Kumpfer, K. L.; DeMar sh, J. K.: "Family environmental and genetic influences on children's future chemical dependency", en Journal of Children in Contemporary Society: Advances in Theory and Applied Research, 18, 1/2 (1985), pp. 117-152.

Kumpfer, K. L.; DeMarsh, J. P.; Child, W. (1989): Strengthening Families Program: Children's Skills Training Curriculum Manual (Prevention Services to Children of Substanceabusing Parents). Social Research Institute, Graduate School of Social Work, University of Utah.

Maguin, E.; Nochajski, T.; Dewit, D.; Macdonald, S.; Safyer, A.; Kumpfer, K. (s/f): The Strengthening Families Program and children of alcoholic's families: effects on parenting and child externalizing behavior. (Researchfunded by National Institute of

[ 102 ] SIPS - PEDAGOGÍA SOCIAL (ISSN-1139-1723) № 14 TERCERAÉPOCA 
Alcohol Abuse and Alcoholism, grant Ro1 AA11647-03).

Orte, C.; GIFES: "Los programas de prevención centrados en la familia: una visión desde la investigación y la práctica", en Revista de la Asociación Proyecto Hombre, 53 (2005a), pp. 14-17.

Orte, C.; GIFES: (2005b). "Una investigació educativa sobre un programa de competència familiar", en M.X. March (dir.) Anuari de l'educació de les Illes Balears 2005. (284-295). Palma: Fundació Guillem Cifre de Colonya.

Orte, C.; GIFES: Volver a ser una familia después de la droga: aplicación de un programa de competencia familiar", en Revista de la Asociación Proyecto Hombre, 57 (2006a), pp. 43-46.

Orte, C.; March, M. X.; Ballester, L.; Touza, C. Oliver, J. L.; Fernández, C.; Mestre, L.: Evaluación de resultados y de proceso del Programa de Competencia Familiar", enRevista de la Asociación Proyecto Hombre, 58 (2006b), pp. $80-83$.

Orte, C,; Touza, C.; (2006): "Avaluació del procés d'execució del Programa de Competència Familiar", en M. X. March (dir.) Anuari de l'educació de les Illes Balears 2006. (352-375). Palma: Fundació Guillem Cifre de Colonya.

Tobler, N. S.; Kumpfer, K. L. (2000): Metaanalysis of effectiveness of family-focused substance abuse prevention programs. Rochville, MD: Report submitted to the Center for Substance Abuse Prevention.

\section{Notas}

${ }^{1}$ Grupo de investigadores formado por miembros del Departamento de Pedagogía y Didácticas Específicas de la Universidad de les Illes Balears. También participan miembros de Proyecto Hombre Baleares y del Hospital Son Dureta (Carmen Orte, Martí X. March, Lluís Ballester, C. Touza, Josep Lluís. Oliver, Belén Pascual, Carmen Fernández, Cristina Fernández y Luisa Mestre).

${ }^{2}$ Con la financiación del Ministerio de Ciencia y Tecnología (BSO2003-08717), FEDER, la Conselleria d'Economia, Hisenda i Innovació y la Conselleria de Salut i Consum del Govern de les Illes Balears.

DiRección de los AUTORes: Carmen Orte Socias, Carmen Touza Garma, Lluis Ballester Brage. Universitat de les Illes Balears. Departamento de Pedagogía y Didácticas Específicas. Cra. Valldemossa, Km.7,5. Campus Universitario. Edificio Guillem Cifre de Colnya. o7122 Palma (Mallorca).

Correo electrónicos: carmen.orte@uib.es; carmen.touza@uibes, lluis.ballester@uib.es

Fecha de recepción del artículo: 23.IX.2006

Fecha de aceptación definitiva: 21.XII.2006 